\title{
STRUCTURED DECOMPOSITIONS FOR MATRIX TRIPLES: SVD-LIKE CONCEPTS FOR STRUCTURED MATRICES
}

\author{
Christian Mehl, Volker Mehrmann and Hongguo Xu
}

Abstract. Canonical forms for matrix triples $(A, G, \hat{G})$, where $A$ is arbitrary rectangular and $G, \hat{G}$ are either real symmetric or skew symmetric, or complex Hermitian or skew Hermitian, are derived. These forms generalize classical singular value decompositions. In [1] a similar canonical form has been obtained for the complex case. In this paper, we provide an alternative proof for the complex case which is based on the construction of a staircase-like form with the help of a structured $Q R$-like decomposition. This approach allows generalization to the real case.

Mathematics subject classification (2000): 15A21, 65F15, 65L80, 65L05.

Keywords and phrases: matrix triples, indefinite inner product, structured SVD, canonical form, Hamiltonian matrix, skew-Hamiltonian matrix.

\section{REFERENCES}

[1] Y. BolschaKov AND B. Reichstein, Unitary equivalence in an indefinite scalar product: an analogue of singular-value decomposition, Linear Algebra Appl., 222 (1995), 155-226.

[2] Y. Bolschakov, C.V.M. van Der Mee, A.C.M. Ran, B. Reichstein, And L. Rodman, Polar decompositions in finite-dimensional indefinite scalar product spaces: general theory, Linear Algebra Appl., 261 (1997), 91-141.

[3] D.Z. Djokovic, J. Patera, P. Winternitz, And H. Zassenhaus, Normal forms of elements of classical real and complex Lie and Jordan algebras, J. Math. Phys., 24 (1983), 1363-1374.

[4] N.M. Dobrovol's 'saya And V.A. Ponomarev, A pair of counter-operators (in Russian), Uspehi Mat. Nauk, 20 (1965), 80-86.

[5] H. Fassbender, D. S. Mackey, N. Mackey, AND H. XU, Hamiltonian square roots of skewHamiltonian matrices, Linear Algebra Appl., 287 (1999), 125-159.

[6] H. Flanders, Elementary divisors of $A B$ and BA, Proc. Amer. Math. Soc., 2 (1951), 871-874.

[7] I. Gohberg, P. Lancaster, And L. Rodman, Matrices and Indefinite Scalar Products, Birkhäuser, Basel, 1983.

[8] I. Gohberg, P. Lancaster, And L. Rodman, Indefinite Linear Algebra and Applications, Birkhäuser, Basel, 2005.

[9] G. H. Golub And C. F. VAn Loan, Matrix Computations, Johns Hopkins University Press, 3rd edition, Baltimore, 1996.

[10] S. HASSI, Matriisin yleisen singulaariarvohajoitelman ominaisuuksia ja tilastollisia sovelluksia, Master's thesis, University of Helsinki, 1984.

[11] S. HASSI, A singular value decomposition of matrices in a space with an indefinite scalar product, Ann. Acad. Sci. Fenn. Ser. A I Math. Dissertationes, 79, 1990.

[12] R. A. Horn And C. R. Johnson, Topics in Matrix Analysis, Cambridge University Press, Cambridge, 1991.

[13] R. A. Horn And D. Merino, Contragredient equivalence: a canonical form and some applications, Linear Algebra Appl., 214 (1995), 43-92.

[14] T. Kapitula, P.G. Kevrekidis, And B. Sandstede, Counting eigenvalues via the Krein signature in infinite-dimensional Hamiltonian systems, Physica D: Nonlinear Phenomena, 195 (2004), 263-282. 
[15] J. Kes AND A.C.M. RAN, On the relation between $X X^{[*]}$ and $X^{[*]} X$ in an indefinite inner product space, Oper. Matrices, 1 (2007), 181-197.

[16] P. Lancaster and L. Rodman, The Algebraic Riccati Equation, Oxford University Press, Oxford, 1995.

[17] P. LANCASTER AND L. Rodman, Canonical forms for Hermitian matrix pairs under strict equivalence and congruence, SIAM Rev., 47, (2005), 407-443.

[18] P. LANCASTER AND L. Rodman, Canonical forms for symmetric/skew symmetric real pairs under strict equivalence and congruence, Linear Algebra Appl., 406 (2005), 1-76.

[19] C. Mehl, V. Mehrmann, And H. XU, Singular-value-like decompositions for complex matrix triples, J. Comput. Appl. Math., to appear, 2009. http://dx.doi.org/10.1016/j.cam.2008.02.017.

[20] C. Mehl, A.C.M. RAN, AND L. Rodman, Polar decompositions of normal operators in indefinite inner product spaces, Oper. Theory Adv. Appl., 162 (2006), 277-292.

[21] V. Mehrmann And H. Xu, Structured Jordan canonical forms for structured matrices that are Hermitian, skew Hermitian or unitary with respect to indefinite inner products, Electron J. Linear Algebra, 5 (1999), 67-103.

[22] J. Patera AND C. Rousseau, Versal deformations of elements of classical Jordan algebras, J. Math. Phys., 24 (1983), 1375-1379.

[23] A. C. M. RAN AND M. WoJTYLAK, Analysis of spectral points of the operators $T^{[*]} T$ and $T T^{[*]}$ in a Krein space, submitted, 2008.

[24] V.V. Sergeichuk, Computation of canonical matrices for chains and cycles of linear mappings, Linear Algebra Appl., 376 (2004), 235-263.

[25] R. C. THOMPSON, The characteristic polynomial of a principal submatrix of a Hermitian pencil, Linear Algebra Appl., 14 (1976), 135-177.

[26] R. C. Thompson, Pencils of complex and real symmetric and skew matrices, Linear Algebra Appl., 147 (1991), 323-371.

[27] H. XU, An SVD-like matrix decomposition and its applications, Linear Algebra Appl., 368 (2003), $1-24$.

[28] H. XU, A numerical method for computing an SVD-like decomposition, SIAM J. Matrix Anal. Appl., 26 (2005), 1058-1082. 